\title{
EDUCATION AND TRAINING Development of a north-west London paracentesis simulation course for core medical trainees
}

\author{
Authors: Georgia Woodfield, ${ }^{\mathrm{A}}$ Luke D Tyson, ${ }^{\mathrm{B}}$ Orhan Orhan ${ }^{\mathrm{C}}$ and Joanna Szram ${ }^{\mathrm{D}}$
}

We designed, implemented and evaluated a near-peer simulation training programme teaching diagnostic and therapeutic abdominal paracentesis to core medical trainees (CMTs). We taught diagnostic and therapeutic abdominal paracentesis to 77 north-west London CMTs over 8 training days over 4 years, 2015 to 2019. The programme was optimised by use of plan, do, study, act (PDSA) cycles and the content was evaluated by anonymous pre- and post-course questionnaires. There was a need for this training; $89 \%$ of participants reported inadequate training opportunities pre-course and only $28 \%$ felt 'confident' or 'very confident' to insert an ascitic drain. Simulation training appears effective when teaching these skills. Having been low in confidence before the course, all participants reported increased confidence after completing the course. Simulation training has been highlighted as a key aspect of the new internal medicine training programme, which replaces CMT. We would recommend using PDSA cycles to implement effective simulation programmes.

KEYWORDS: simulation, paracentesis, education

DOI: $10.7861 /$ fhj.2020-0153

\section{Introduction}

Core medical training (now internal medicine training) was a key period of skill and knowledge acquisition for junior doctors prior to higher specialty training. Core medical trainees (CMTs), like current internal medicine trainees (IMTs), needed

Authors: ${ }^{A}$ gastroenterology specialty registrar, West Middlesex University Hospital, London, UK and clinical research fellow, Imperial College London, London, UK; ${ }^{B}$ senior medicine teaching fellow and gastroenterology specialty registrar, London North West University Healthcare NHS Trust, London, UK and honorary clinical research fellow, Imperial College London, London, UK;

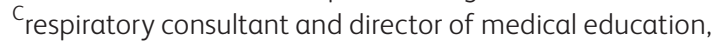
Chelsea and Westminster Hospital, London, UK; ${ }^{\mathrm{D}}$ consultant in occupational lung disease, Royal Brompton Hospital, London, UK, deputy postgraduate dean and clinical lead for physician specialty recruitment, Health Education England South London, London, UK and honorary senior lecturer, National Heart and Lung Institute, London, UK to be competent in numerous practical procedures before completion. ${ }^{1}$ CMTs performed supervised procedures until deemed competent, through direct observation of procedural skills (DOPS) assessments. Clinical independence in diagnostic abdominal paracentesis was mandatory, and therapeutic abdominal paracentesis desirable. However, CMTs consistently reported difficulties with procedural skills development. Service provision and the adoption of shift-working patterns was perceived as limiting the time available for supervised practice., ${ }^{2,3}$

\section{Problem description}

Anecdotally, CMTs at our trusts reported difficulties gaining procedural competencies due to lack of opportunity. This correlated with CMTs nationally. ${ }^{3}$

\section{Available knowledge}

Simulation-based teaching is a way of training psychomotor and perceptual skills, without the time pressures and patient safety concerns inherent to real-life practice. At the time, simulation was already widely used in postgraduate medical training (for example, during Resuscitation Council UK's life support courses). Simulation-training had been shown to be effective when teaching practical skills. Laparoscopic surgery had one of the largest research bases; studies demonstrated a positive effect of virtual reality simulation training on the learning curve of surgical trainees. ${ }^{4-9}$ In gastroenterology, the American Council for Graduate Medical Education had mandated the incorporation of procedural simulation training into US gastroenterology fellowship programmes. ${ }^{10}$

\section{Rationale}

Our educational needs assessment of our CMTs revealed a lack of skills acquisition for abdominal paracentesis. We felt a simulation-based approach would be a logical way to tackle this issue. While we accepted it would be challenging to assess how our intervention affected patient outcomes, we felt that this approach had the potential to improve the CMTs' knowledge and skills. Simulation was accepted as a valid method of training. ${ }^{11}$

We planned teaching around the key features essential for abdominal paracentesis but did not recreate all the real-life elements. This approach does not reduce the educational impact of simulation. ${ }^{11}$ Evidence suggests the needs of a learner can be better met when teachers and learners are at a similar stage. ${ }^{12-14}$ Hence, we introduced near-peer learning. 
Aim

We aimed to design, implement and evaluate a north-west London training programme for CMTs, teaching diagnostic and therapeutic abdominal paracentesis, run by specialist medical trainees experienced in these procedures.

\section{Objectives}

We had four core objectives.

$>$ Evaluate near-peer simulation training as a method of teaching abdominal paracentesis, using participant feedback and realtime behavioural observation.

> Optimise the teaching programme by evaluating and improving it at each stage, using plan, do, study, act (PDSA) cycles.

> Disseminate our experiences to others in the field to improve collective practice.

> Integrate our programme into the centrally organised teaching programme offered to north-west London CMTs.

\section{Methods}

We conceived the idea in 2014 as north-west London medical trainees. The project was supported by the then director of medical education at the Royal Brompton Hospital. We won a bid for funding from Health Education North West London and purchased a paracentesis simulation model and associated equipment in early 2015. Other equipment was sourced from in-hospital supplies. We purchased a further model after a second successful funding bid to Health Education North West London in October 2016. The course was run in the clinical skills centre at the Royal Brompton Hospital.

\section{Intervention}

A pilot programme was designed for eight CMTs per 2-hour session. Each session was composed of two parts.

> Introductory PowerPoint presentation to explain theory, based on British Society of Gastroenterology and European Association for the Study of the Liver guidelines for managing ascites, including a video demonstration of abdominal paracentesis. ${ }^{15,16}$

> Practical skills stations for diagnostic and therapeutic paracentesis, using ascitic abdomen simulation models. For the skills stations, the CMTs were divided into two groups who swapped between parallel stations. Both procedures were demonstrated by tutors initially. At one station, CMTs performed diagnostic paracentesis then discussed case studies including ascitic fluid lab analysis results. At the parallel station CMTs performed therapeutic paracentesis on a purpose-built paracentesis model.

\section{Evaluation of the Intervention}

A pre-course questionnaire (supplementary material S1) explored prior knowledge of abdominal paracentesis, participant perceptions of previous learning opportunities, confidence in this skill, confidence identifying the correct patients and explaining the procedure, and confidence in dealing with complications. This enabled us to assess the learning needs of the CMTs and to evaluate the impact of our course when contrasted with the postcourse questionnaire.

The post-course questionnaire (supplementary material S2) evaluated the course in terms of content, length, relevance, teaching style and perceived confidence in the skill after teaching. This allowed us to evaluate our teaching and course design.

In line with quality improvement principles, we used PDSA cycles to evaluate each teaching session and improve our course, as detailed.

\section{Analysis}

Participant confidence scores were analysed using MS Excel (Microsoft, Redmond, USA) and SPSS Statistics V27 (IBM, Armronk, USA). Paired pre- and post-course ordinal data (for example, level of perceived confidence) was compared by Wilcoxon signed-rank test. A $p<0.05$ was considered significant. Free text comments were recorded from the questionnaires, but verbal feedback was not included in the analysis as it could be subject to bias given its lack of anonymity.

\section{Ethical considerations}

CMTs attended the course after an advert was circulated to Royal Brompton and Harefield NHS Foundation Trust CMTs, and later to all north-west London CMTs. A possible inequality is that other CMTs missed out on the opportunity to attend this training, potentially putting them at a disadvantage. We hope to make the course available to all IMTs in London in the future but were obliged to pilot it for a small number of trainees first. It was not intended to replace other opportunities available to CMTs for example, local teaching and DOPS assessment. Questionnaires were optional and anonymous. Trainees were informed on arrival that the purpose of the questionnaires was to help develop the course, and to allow us to disseminate good practice. As an educational service improvement project, formal ethics board approval was not required.

\section{Timeline}

We considered each iteration of the course as a separate PDSA cycle: we analysed each course during a post-course meeting and agreed improvements; we implemented these changes during the next course; we evaluated the consequences of our changes; and we then determined how we could improve the next course.

We conceived the programme in December 2014. We secured funding and a venue, agreed the course structure and designed a pre- and post-course questionnaire as described. The first session was in April 2015 with eight CMTs. The pre-course questionnaire highlighted that CMTs had inadequate experience of paracentesis during their rotations. This confirmed the need for our course. The post course questionnaire gave excellent feedback on the structure of the course. We refined the case-based theory element and altered the introductory slides to cover common queries. We presented our methodology for setting up a simulation training programme as a poster at the Developing Health Educators of the Future conference in early July 2015. This provided opportunities to network with other educators and to get advice about our setup and methodology. Following the pilot, our course was added to the central teaching programme offered to north-west London CMTs and advertised centrally. 
The second session was in July 2015. We found that our paracentesis model (Paracentesis and Thoracentesis Procedure Trunk, Sawbones) had become difficult to pierce effectively with repeated use. We acted on this by investigating alternatives and found that some educators were using home-made models. We made two models from $1 \mathrm{~L}$ bags of crystalloid tightly wrapped in absorbent pads and plastic/rubber material, to imitate skin. We used these at the third session in January 2016. These models were an improvement on the previous model, the tight wrapping made the 'ascites' tense and insertion felt more realistic with good flash-back of fluid into the needle. However, they required careful management, as fluid leaked regularly onto the table. We purchased a new model (Ultrasound Paracentesis Trainer, Simulab).

We held the fourth session in May 2016. We had previously observed that a significant proportion of CMTs who signed-up did not attend on the day, without explanation as to why. We decided to use Eventbrite for recruitment as this was the platform preferred by the local education and training board. The new model was superior than the previous model as it felt more realistic during drain insertion, and it did not leak like the homemade models. It was also ultrasound compatible.

Despite using Eventbrite, attendance on the day remained a concern. The capacity of each of the initial sessions was only eight participants. Each session was over-subscribed, and it was frustrating to turn down applicants when some of the CMTs who had signed-up did not attend on the day. We wanted to increase capacity to accommodate more CMTs, accepting that some would not attend. Hence, we planned a larger session with capacity for 24 CMTs. To allow this, we combined our session with sessions on other clinical skills (joint aspiration and non-invasive ventilation) run by specialists in those areas. We ran three parallel 2-hour sessions with capacity for eight CMTs in each group, rotating between sessions. The fifth session took place in January 2017.

Attendance at the fifth session was again low (11 out of the 24 CMTs who had signed up attended), but this was still our biggest group to date. We ran the combined course on three further occasions: November 2017, July 2018, and January 2019. We sent frequent reminder emails, highlighting the waiting list, and asking for notice if participants who had signed up were unable to attend. Despite these measures, we saw variable attendance: 22/28 in November 2017,
$7 / 20$ in July 2018 , and $13 / 20$ in January 2019 . For the next course, we are planning to ask for a small monetary deposit, refundable on attendance, and we hope this may improve attendance further.

Table 1 summarises the main problems that we encountered during the development of the course and our response to these.

\section{Results}

Seventy-seven participants attended the eight sessions: 53 CMTs attended the four combined course sessions and 24 CMTs attended the initial four sessions. Responses are amalgamated.

\section{Pre-course}

In total, 63 CMTs completed the pre-course questionnaire. Eighty-nine per cent reported inadequate opportunities to learn how to insert an ascitic drain and $70 \%$ had worries or concerns about inserting ascitic drains. However, all CMTs had at least some confidence in knowing when insertion of a drain is required, and how to explain it to a patient (100\% scored 'some confidence', 'confident' or 'very confident').

Free-text comments included:

Not enough experience/opportunity especially if you don't have a gastro job on CMT.

I am worried because a registrar training me previously had experienced bowel perforation in a patient being drained who subsequently died.

The free-text reasons for CMTs wanting to come on the course included:

I hope to gain skills and knowledge.

Ability to insert ascitic drains with confidence and supervision.

Learn basics about inserting ascitic drains and dealing with complications.

\section{Post-course}

There was an increase in respondent confidence in inserting ascitic drains from pre- to post-course (Fig 1a), and in managing potential complications (Fig 1b). Analysis of the respondents who

\section{Table 1. Problems identified during teaching sessions, and how they were addressed}

\begin{tabular}{|c|c|c|c|c|}
\hline Problem & Intervention & Result & Solved? & Next step \\
\hline $\begin{array}{l}\text { Paracentesis model } \\
\text { leaking after repeated use } \\
\text { (Sawbones) and not very } \\
\text { realistic }\end{array}$ & $\begin{array}{l}\text { Made our own ascitic } \\
\text { abdomens using bags } \\
\text { of normal saline and } \\
\text { incontinence pads (single } \\
\text { session use only) }\end{array}$ & $\begin{array}{l}\text { Excellent feedback } \\
\text { from students: } \\
\text { more realistic feel }\end{array}$ & $\begin{array}{l}\text { Yes - problem of } \\
\text { realism solved, } \\
\text { however, messy and } \\
\text { single use only }\end{array}$ & $\begin{array}{l}\text { Purchase of new model } \\
\text { (Simulab): much more } \\
\text { realistic and able to be used } \\
\text { again after one session } \\
\text { (multiple use), the longevity } \\
\text { yet to be seen }\end{array}$ \\
\hline $\begin{array}{l}\text { Insufficient course } \\
\text { capacity: multiple core } \\
\text { medical trainees on a } \\
\text { waiting list each course }\end{array}$ & $\begin{array}{l}\text { Course combined with other } \\
\text { skills sessions to expand total } \\
\text { capacity }\end{array}$ & $\begin{array}{l}\text { Absolute numbers } \\
\text { of students } \\
\text { attending course } \\
\text { increased }\end{array}$ & $\begin{array}{l}\text { Partially - pre- } \\
\text { course demand still } \\
\text { exceeded capacity }\end{array}$ & $\begin{array}{l}\text { Reduce participants failing } \\
\text { to attend on the day to } \\
\text { optimise use of capacity }\end{array}$ \\
\hline $\begin{array}{l}\text { Numerous core medical } \\
\text { trainees failed to attend on } \\
\text { the day }\end{array}$ & $\begin{array}{l}\text { Used Eventbrite, a sign-up } \\
\text { site familiar to trainees used } \\
\text { by the LETB }\end{array}$ & $\begin{array}{l}\text { Attendance still } \\
\text { suboptimal but has } \\
\text { slowly improved } \\
\text { over time }\end{array}$ & No & $\begin{array}{l}\text { Consider a monetary } \\
\text { deposit, to be returned upon } \\
\text { attendance }\end{array}$ \\
\hline
\end{tabular}

$\mathrm{LETB}=$ local education and training board 


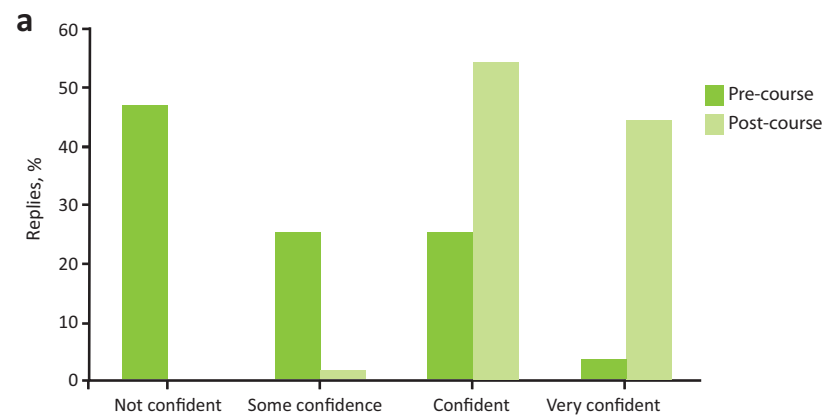

b

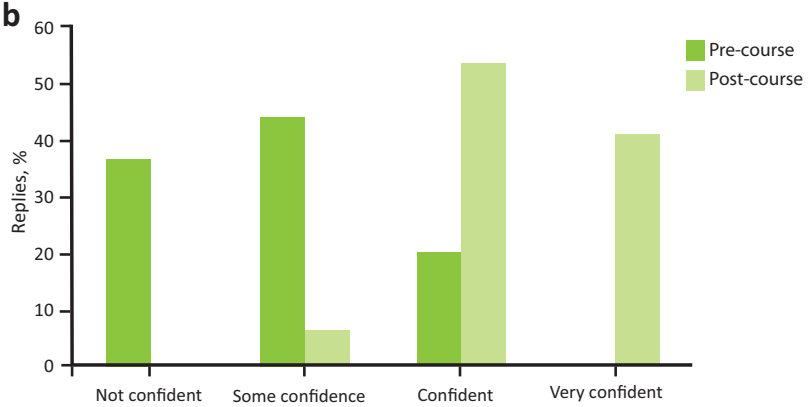

Fig 1. Questionnaire responses assessing pre- and post-course participant confidence around ascitic drains. a) Question: 'How confident do you feel about inserting ascitic drains?' Pre-course, $n=60$; post-course, $n=63$. b) Question: 'How confident do you feel about managing any potential complications?' Pre-course, $n=55$; post-course, $n=64$.

returned paired responses demonstrated a significant increase in confidence inserting ascitic drains ( 60 pairs; $Z=-6.86 ; p<0.001$ ) and managing complications ( 55 pairs; $Z=-6.683 ; p<0.001$ ).

Fifty-five of the 77 participants fully completed both pre- and post-course questionnaires.

$>100 \%$ of these participants felt more confident than they had before the training.

$>100 \%$ felt that the training would have a positive impact on their future practice.

$>100 \%$ of trainees classed the introductory slides, the diagnostic paracentesis station, the therapeutic paracentesis station, the teaching standard and the overall session as 'very useful'.

$>100 \%$ of participants felt that the session covered the topics adequately and was an appropriate length.

> Twenty-two participants did not fully complete both questionnaires, which may potentially bias the results towards those who had a positive experience as those who had a negative experience may have been less likely to complete the questionnaires.

Free-text comments responding to the question 'did you learn anything new' included:

Techniques for ascitic tap and drainage.

Practical skills technique for insertion of drain.

My technique improved and I learnt about the indications of interpretation of results.

General free-text comments included:
Excellent, great session! Very useful session especially the ascitic drain insertion and [non-invasive ventilation] station.

Excellent session, thank you!

Thank you, great session!

Awesome! One of the best prepared in terms of information and kit!

Do it for everyone!

Very helpful, thank you. Could also include demo with ultrasound.

Very useful session relevant to CMT curriculum. Excellent teaching, if [ultrasound] was available would be useful

Needs to be offered to all CMT trainees.

\section{Summary}

We used near-peer simulation training to teach diagnostic and therapeutic abdominal paracentesis to 77 north-west London CMTs across 8 separate training days over 4 years. Participant confidence significantly improved by the end of the course, and participants reported that the course would have a positive impact on their future practice.

\section{Discussion}

Performing paracentesis on a simulated ascitic abdomen will never be completely true-to-life, and there are clear limitations. Firstly, the rubber of the simulator is tougher than human skin so the haptic feedback and feeling of drain insertion is imperfect. Secondly, communication is simulated with the trainer pretending to be the patient. However, this lack of realism becomes less of a limitation when simulation is used as a preparation or revision tool rather than a replacement for real practice. In medicine, simulation is generally used to represent reality; much time and money is spent in striving to achieve this realism (for example, making manikins as human as possible). This is based on the belief that reality is the gold standard, and that simulation should aim to prepare the learner for that eventuality. However, simulation can also be a learning resource. Trainees may even be more likely to remember details of a simulated procedure, as the lack of time pressure and safe environment may allow them to process the skills that they are learning. ${ }^{3-5}$

Simulation also allows for errors to be made in a safe environment. Errors are a positive part of the learning process: students may be more likely to reflect on the theory and retain the information if they have made an error themselves. Thinking through the process enforces Kolb's learning cycle for long-term retention of knowledge. ${ }^{17}$

We used confidence and perceptions of learning as a measurement of success. This may not necessarily translate into good clinical outcomes. While it can be difficult to prove that simulation has a positive effect on clinical practice, the use of simulator technology to teach standardised procedures in anaesthesia has been proven to be effective. ${ }^{18-21}$ A further criticism of simulation is that there can be a large gap between having knowledge and applying that knowledge in real life. ${ }^{22}$ One way of measuring the application of knowledge is by DOPS assessment, yet there is little evidence that DOPS assessments improve patient outcomes. Simulation has been shown to be effective in other 
industries, and it is perhaps intuitive that it improves preparation for real-life encounters. ${ }^{23}$ Adverse events due to medical errors account for much morbidity and mortality, therefore some action needs to be taken to address this, and simulation seems a sensible, and relatively risk-free, way to proceed. ${ }^{11}$

Regarding our programme, attendance on the day remained an issue despite all sessions being booked to capacity. Reasons for non-attendance were not assessed in our study but this is an important future consideration given the need to maximise the impact of any given teaching session. It is unclear why attendance varied between sessions but common barriers (for example service provision) may have impacted attendance. ${ }^{3}$ However, although the courses did not reach capacity, over twice as many CMTs attended the four combined courses than the initial four courses teaching just paracentesis, justifying the change in format.

Most trainers on this course were medical trainees themselves (largely registrar level), yet all candidates gave the maximum score for the standard of teaching for all sessions. We feel that this supports the use of near-peer learning. There may be abstruse advantages to this, as trainees asked questions surrounding job applications, interviews, and general advice, which the trainers were well placed to answer. Trainee-led projects also help to engage consultants of the future in training and education and help to build educational capability. For example, after the initial pilot, our course was added to the central teaching programme for north-west London CMTs.

IMT replaced CMT in August 2019. IMT year-2 trainees require the same competencies as CMT. IMTs have mandatory rotations in intensive care and geriatric medicine, potentially reducing opportunities to rotate through specialties (such as gastroenterology and respiratory medicine) where they may have greater opportunity to practice mandatory procedures. ${ }^{24}$ Consequently, simulation training programmes are likely to become increasingly important for skills development. Although we describe here the development of a paracentesis simulation training programme, we believe our conclusions are generalisable to other core procedures such as thoracentesis or central venous catheterisation.

We feel that using PDSA cycles in addition to questionnaire responses allowed us to rapidly optimise our models and course structure. We would recommend this strategy for colleagues designing future training programmes, whether for clinical skills or for alternative competencies. We hope that our success demonstrates that people support good ideas and that it is possible to implement them, even as a junior doctor.

\section{Conclusion}

We designed and implemented an abdominal paracentesis simulation training programme within a London deanery using near-peer learning. Using simulation appears to be an effective method of teaching this skill; candidate feedback was positive regarding the content and relevance of the sessions, and confidence scores universally rose after teaching. The perception of the CMTs was that the course was 'very useful'. As course facilitators, we felt that trainees were competent at performing diagnostic and therapeutic paracentesis in a simulated setting after the teaching. Our next challenge is to expand the capacity of this programme such that the majority of IMTs in our region can attend.

\section{Supplementary material}

Additional supplementary material may be found in the online version of this article at www.rcpjournals.org/fhj:

S1 - Questionnaire prior to paracentesis practical skills course.

S2 - Questionnaire after paracentesis practical skills course.

\section{Conflicts of interest}

Jo Szram is on the editorial board of Future Healthcare Journal.

\section{References}

1 Joint Royal Colleges of Physicians Training Board. Speciality training curriculum for core medical training. JRCPTB, 2013. www. jrcptb.org.uk/sites/default/files/2009\% 20CMT\% 20Curriculum \% 20 \% 28amendments \% 202013\% 29\% 20100516.pdf

2 Amery C, Griffin A. Exploring communities of practice in the NHS: A core medical trainee experience. FHJ 2020;7:e1-5.

3 Tasker F, Newbery N, Burr B, Goddard AF. Survey of core medical trainees in the United Kingdom 2013 - inconsistencies in training experience and competing with service demands. Clin Med 2014:14:149-56.

4 Aggarwal R, Ward J, Balasundaram I et al. Proving the effectiveness of virtual reality simulation for training in laparoscopic surgery. Ann Surg 2007:246:771-9.

5 Ahlberg G, Enochsson L, Gallagher AG et al. Proficiency-based virtual reality training significantly reduces the error rate for residents during their first 10 laparoscopic cholecystectomies. Am J Surg 2007;193:797-804.

6 Grantcharov TP, Kristiansen VB, Bendix ] et al. Randomized clinical trial of virtual reality simulation for laparoscopic skills training. $\mathrm{Br}$ ] Surg 2004;91:146-50.

7 Larsen CR1, Soerensen JL, Grantcharov TP et al. Effect of virtual reality training on laparoscopic surgery: randomised controlled trial. BMJ 2009;338:b1802.

8 Seymour NE, Gallagher AG, Roman SA et al. Virtual reality training improves operating room performance: results of a randomized, double-blinded study. Ann Surg 2002;236:458-63.

9 Aggarwal R, Crochet P, Dias A et al. Development of a virtual reality training curriculum for laparoscopic cholecystectomy. $\mathrm{Br}$ ] Surg 2009;96:1086-93.

10 Accreditation Council for Graduate Medical Education. ACGME program requirements for graduate medical education in internal medicine. ACGME, 2016:2. www.msm.edu/Education/GME/ IMResidencyProgram/140_internal_medicine_2016.pdf

11 Kohn LT, Corrigan JM, Donaldson MS. To err is human: building a safer health system. Washington: National Academy Press, 1990.

12 Rettedal A. When reality is difficult to simulate. European Journal of Anaesthesiology 2000;17:515-26.

13 Woodfield G, O'Sullivan G. Clinical teaching fellows, everyone's a winner. Clin Teach 2014;11:136-40.

14 Topping $\mathrm{K}$. The effectiveness of peer tutoring in further and higher education: a typology and review of the literature. Higher Education 1996;32:321-45.

15 Moore KP, Aithal GP. Guidelines on the management of ascites in cirrhosis. Gut 2006;55 Suppl 6:vi1-12.

16 European Associate for the Study of the Liver. EASL clinical practice guidelines on the management of ascites, spontaneous bacterial peritonitis, and hepatorenal syndrome in cirrhosis. Journal of Hepatology 2010;53:397-417.

17 Kolb DA. Experiential learning: Experience as the source of learning and development. Englewood Cliffs: Prentice Hall, 1984.

18 Cummins R. Textbook of advanced cardiac life support. American Heart Association, 1994. 
19 Gaba D, Maxwell M, DeAnda A. Anesthetic mishaps: Breaking the chain of accident evolution. Anesthesiology 1987;66:670-6.

20 Monk S, Baldering H, Vollmer J, Buggenhagen H, Heinrichs W. Patientensimulation. Notf Rettungsm 1999;2:297-306.

21 Rettedal A, Stale F, Ragna K, Petter L. PatSim: simulator for practising anaesthesia and intensive care. Development and observations. International Journal of Clinical Monitoring and Computing 1996;13:147-52.

22 Nimmo G, Glavin R, Baruch M, Hartley S. Teaching the management of medical emergencies. European Journal of Anaesthesiology 2000;17:520-1.

23 Kuhnigk $\mathrm{H}$. From mega-code training to simulator session. What is the benefit for the patient? European Journal of Anaesthesiology 2000;17:517.
24 Joint Royal Colleges of Physicians Training Board. Curriculum for internal medicine: Stage 1 training. JRCPTB, 2019. www.jrcptb.org uk/sites/default/files/IM_Curriculum_Sept2519.pdf.

Address for correspondence: Dr Luke Tyson, Undergraduate Department, London North West University Healthcare NHS Trust, Northwick Park Hospital, Watford Road, Harrow, London HA1 3U], UK.

Email: luke.tyson@nhs.net

Twitter: @lungsatwork 